\title{
Analysis and Design for Class-E ZVS DC-DC Converters
}

\author{
Xiang Zhang ${ }^{1, ~ a, ~ J i n g ~ C h u n ~ H u a n g ~}{ }^{2, b}$ and Jian Xiao ${ }^{3, c}$ \\ ${ }^{1}$ School of Electrical Engineering, Southwest Jiaotong University, Chengdu, China \\ ${ }^{2}$ School of Electrical Engineering, Southwest Jiaotong University, Chengdu, China \\ ${ }^{3}$ School of Electrical Engineering, Southwest Jiaotong University, Chengdu, China \\ amaglevzx@163.com, bjchuang@home.swjtu.edu.cn, cjxiao@home.swjtu.edu.cn
}

Keywords: DC/DC converters, Class-E inverters, Resonant rectifiers, Zero voltage switch (ZVS), Design equations.

\begin{abstract}
The paper introduces the detailed derivation and design procedure for class-E DC-DC converters operated at very high frequency. Design formulae of the converter are derived. The converter features zero or low $d v / d t$ at the turn-on and turn-off instants of the switch in the inverter and the diode in the rectifier. Hence, it greatly alleviates the adverse effects caused by the parasitic inductance and capacitance from circuits and components. The circuit of the converter is typically non-linear and very complicated. Therefore, it is very difficult or impossible to analyze the converter directly, and an appropriate simplification analysis is required. Because the resonant frequency of the rectifier is very near to the switching frequency of the inverter and the $L C$ tank of the rectifier has an enough high $Q$, these two conditions guarantee the converter can be divided into two independent parts, which do not influence each other. Based on that, the inverter and the rectifier can be analyzed and designed respectively. To verify the proposed converter and design method, a converter operated at 50 $\mathrm{MHz}$ is designed and simulated. The simulation waveforms are in close agreement with the results from the analysis, and validate the proposed design method is feasible and effective.
\end{abstract}

\section{Introduction}

Because of the developments of electronic devices and circuits, it is possible to switch and control DC/DC power converters at very high switching frequency. High switching frequencies improve greatly the output performance of power supply system. Operated at high switching frequencies, the parameters of passive storage parts in circuits are very small, even, these passive components are replaced with the parasitic inductance or capacitance of other components. Therefore, the dimensions of power converters are reduced significantly [1], the power density of power converters arises, accordingly. The cost also decreases much. Besides, high switching frequencies make power converters respond rapidly. Unfortunately, high switching frequencies bring in a serious problem, switching loss rises sharply. Switching loss grows with the increase of switching frequency, and it is in proportion to the value of switching frequency, namely, the higher switching frequency increases, the more switching loss are produced. ZVS (zero voltage switch) and ZCS (zero current switch) can solve this difficult issue very well [2], [3]. In general, resonant topology can be employed to implement soft switching. Taking advantage of series or parallel resonant circuits, the voltage across switch components or the current through switch components becomes zero at switching instants. 


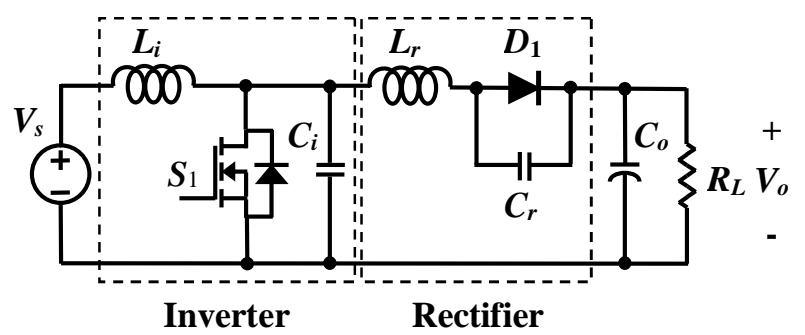

Fig. 1 Schematic of class-E VHF DC/DC converters

A class-E VHF DC/DC converter will be studied in this paper and its circuit topology is shown in Fig. 1. The converter consists of an inverter and a rectifier. The inverter is a Class-E inverter, which is the most efficient inverter known so far [2]. In addition, ZVS is implemented with a resonant networks. The rectifier is a resonant rectifier, which features zero or low $d v / d t$ [4], [5]. For generic half-wave rectifiers and full-bridge rectifiers, switch components suffer from undesirable voltage spike and noise caused by the parasitic oscillation which comes from parasitic inductance and the junction capacitance of the rectifier diode [6]. Especially, the parasitic oscillation becomes very severe at very high switching frequencies. The rectifier designed in this paper reduces greatly the parasitic resonance in circuits and the voltage stress of switch components decreases highly [6]-[9]. Besides, the focus of this paper differs from that of previous papers [6]-[13], the derivation and design procedure of the inverter with ZVS and the resonant rectifier are presented in detail, design equations are derived as well.

In section 2, the converter is analyzed in detail and a proper simplification method is given. In section 3, a design method of the inverter based on ZVS is presented and illustrated. In section 4, a design method of the resonant rectifier is provided and expounded. In section 5, a design example and its simulation results validate the proposed design equations in this paper. Finally, in section 6 , the paper is concluded.

\section{Analysis and Description of Converter}

The proposed converter is a typical nonlinear dynamic system which consists of $L_{i}, C_{i}, L_{r}$ and $C_{r}$ acting as energy storage and conversion elements, a switch $S_{1}$ operating the inverter and a diode $D_{1}$ employed as a rectification element. A pulse series with 50\% duty cycle drives $S_{1}$. According to the switch status of $S_{1}$ and $D_{1}$, the converter works in four cases: (1) $S_{1}$ and $D_{1}$ are turned on, (2) $S_{1}$ is turned on and $D_{1}$ is turned off, (3) $S_{1}$ is turned off and $D_{1}$ is turned on, and (4) $S_{1}$ and $D_{1}$ are turned off.

The mathematical description of the converter is very complicated. Furthermore, the switching time of the diode $D_{1}$ is unknown [1]. Therefore, the solution of the dynamic mathematical model becomes pretty difficult, even, there are no closed-form equations to directly represent the solutions. Although there are some ways which can solve the model and give a detailed design scheme, the solution process will expend too much time and it is difficult to examine design results. No doubt, it is necessary to simplify the system model and mathematical description. Fortunately, because the converter employs a resonant rectifier, there is an effective approach to reduce its model. The converter is operated at a high frequency which is very near to the resonant frequency of the $L C$ tank made up of $L_{r}$ and $C_{r}$. Moreover, the tank has an enough high $Q$. The two conditions ensure that the converter can be divided into two parts, a inverter and a rectifier which don't influence each other. Accordingly, it is possible to analyze and design the inverter and the rectifier respectively. 
For the converter, the output of the inverter is the input of the rectifier. Therefore, as the output of the inverter, the entire rectifier can be regarded as a current source, which consists of a sinusoidal current source and a DC current source. While for the rectifier, the entire inverter is regarded as a sinusoidal voltage source. The equivalent inverter and rectifier are shown in Fig. 2. Because the current through $L_{r}$ and the voltage across $C_{i}$ are not purely sinusoidal, there is a certain degree of error but it is small enough to the converter. So the error caused by the simplification will be eliminated after small corrections in a circuit simulation [1].

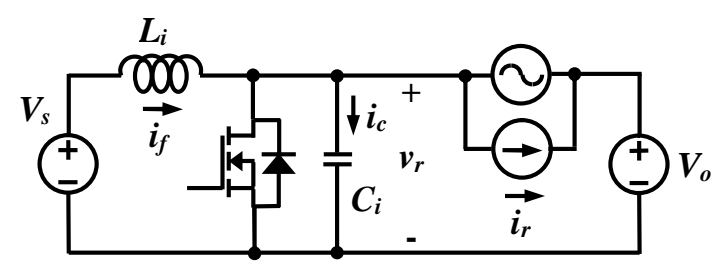

(a) Equivalent inverters.

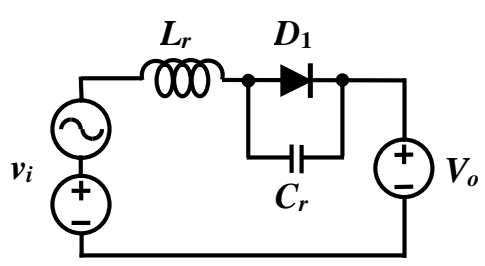

(b) Equivalent rectifiers.

Fig. 2 Equivalent inverters and rectifiers.

\section{Design Method of Resonant Inverter}

For the inverter, the below analysis is based on two conditions. One is an assumption of output current source, the other is related to duty cycle. As the output current, $i_{r}(t)$ can be defined as follows:

$$
i_{r}(t)=I_{m 1} \sin \left(\omega_{s} t+\varphi_{1}\right)+\frac{P_{o}}{V_{o}}
$$

where, $P_{o}$ is the output power of the converter, $V_{o}$ is the output voltage of the converter, $\omega_{s}$ is the operating frequency which is equal to the switching frequency of the converter, $I_{m 1}$ and $\varphi_{1}$ represent the fundamental amplitude and phase of the current through $L_{r}$, respectively. The duty cycle can be set to an arbitrary value from 0 to 1 . Generally, it is set to 0.5 . Based on that, a pulse series with the duty cycle of a half is chosen to design the converter in this paper. Besides, the switch $S_{1}$ is turned off during the time period $0 \leq t<T_{s} / 2$, and it is turned on during the time period $T_{s} / 2 \leq t<T_{s}$.

At the instant $t=0$, the switch $S_{1}$ begins to be turned off. While the switch is off, a circuit equation is derived from Fig. 2(a) as follows:

$$
i_{f}=i_{r}+i_{c}
$$

For the inductor $L_{i}$, the voltage across it and the current through it must meet a constraint, which is

$$
V_{s}-v_{r}(t)=L_{i} \frac{d i_{f}(t)}{d t}
$$

While for the capacitor $C_{i}$, the constraint between the voltage across it and the current through it is,

$$
i_{c}(t)=C_{i} \frac{d v_{r}(t)}{d t}
$$

Then Eq. 3 and Eq. 4 are substituted into Eq. 2, a second-order differential equation for the voltage $v_{r}(t)$ can be obtained as follows:

$$
L_{i} C_{i} \frac{d^{2} v_{r}(t)}{d t^{2}}+v_{r}(t)=V_{s}-\omega_{s} I_{m 1} L_{i} \cos \left(\omega_{s} t+\varphi_{1}\right)
$$

For Eq. 5, its solution can be represented as

$$
v_{r}(t)=A_{1} \sin \left(\omega_{i} t\right)+A_{2} \cos \left(\omega_{i} t\right)+V_{s}+\frac{\omega_{s} L_{i} I_{m 1}}{\omega_{s}^{2} L_{i} C_{i}-1} \cos \left(\omega_{s} t+\varphi_{1}\right)
$$


where

$$
\omega_{i}=\frac{1}{\sqrt{L_{i} C_{i}}},
$$

$A_{1}$ and $A_{2}$ are unknown and constant. Thus, two initial conditions are required to solve these two constants. As the switch $S_{1}$ is turned off at $t=0$, the voltage across the capacitor $C_{i}$ is known and its value is zero. Apart from that, a ZVS condition can be made use of. The voltage of the capacitor $C_{i}$ at $t$ $=T_{s} / 2$ is zero. These initial conditions are represented as

$$
\begin{aligned}
& A_{2}+V_{s}+\frac{\omega_{s} L_{i} I_{m 1}}{\omega_{s}^{2} L_{i} C_{i}-1} \cos \varphi_{1}=0 \\
& A_{1} \sin \left(\frac{\omega_{i} T_{s}}{2}\right)+A_{2} \cos \left(\frac{\omega_{i} T_{s}}{2}\right)+V_{s}-\frac{\omega_{s} L_{i} I_{m 1}}{\omega_{s}^{2} L_{i} C_{i}-1} \cos \varphi_{1}=0
\end{aligned}
$$

So, $A_{1}$ and $A_{2}$ are figured out. At the instant $t=T_{s} / 2$, the switch $S_{1}$ begins to be turned on. While the switch is on, the voltage across the capacitor $C_{i}$ is always zero. Finally, the voltage $v_{r}(t)$ can be described with the following equation:

$$
v_{r}(t)= \begin{cases}\frac{Z_{i}}{\sin \frac{\pi \omega_{i}}{\omega_{s}}}\left[\frac{\omega_{s}}{\omega_{i}} r_{i} I_{m 1} \cos \varphi_{1}\left(1+\cos \frac{\pi \omega_{i}}{\omega_{s}}\right)+\frac{V_{s}}{Z_{i}}\left(\cos \frac{\pi \omega_{i}}{\omega_{s}}-1\right)\right] \sin \left(\omega_{i} t\right) & \\ -\left(\frac{Z_{i} \omega_{s}}{\omega_{i}} r_{i} I_{m 1} \cos \varphi_{1}+V_{s}\right) \cos \left(\omega_{i} t\right)+V_{s}+\frac{Z_{i} \omega_{s}}{\omega_{i}} r_{i} I_{m 1} \cos \left(\omega_{s} t+\varphi_{1}\right), & 0 \leq t<\frac{T_{s}}{2} \\ 0, & \frac{T_{s}}{2} \leq t<T_{s} .\end{cases}
$$

where

$$
Z_{i}=\sqrt{\frac{L_{i}}{C_{i}}}, \text { and } r_{i}=\frac{1}{\left(\omega_{s} / \omega_{i}\right)^{2}-1} .
$$

There are four variables in Eq. 9, and they are $\varphi_{1}, I_{m 1}, L_{i}$ and $C_{i}$ respectively. Other design conditions or constraints are required to solve these unknown parameters.

From Eq. 3, the current through $L_{i}$ can be represented with the following form:

$$
i_{f}(t)=\int_{0}^{t}\left[V_{s}-v_{r}(t)\right] d t+i_{f}(0)
$$

Eq. 9 is substituted into Eq. 10, the current through $L_{i}$ is represented as follows:

$$
i_{f}(t)=\left\{\begin{array}{lr}
\frac{\omega_{s} r_{i} I_{m 1} \cos \varphi_{1}\left(1+\cos \frac{\pi \omega_{i}}{\omega_{s}}\right)\left[\cos \left(\omega_{i} t\right)-1\right]}{\omega_{i} \sin \frac{\pi \omega_{i}}{\omega_{s}}}+\frac{V_{s}\left(\cos \frac{\pi \omega_{i}}{\omega_{s}}-1\right)\left[\cos \left(\omega_{i} t\right)-1\right]}{\omega_{i} L_{i} \sin \frac{\pi \omega_{i}}{\omega_{s}}} \\
+r_{i} I_{m 1}\left[\sin \varphi_{1}-\sin \left(\omega_{s} t+\varphi_{1}\right)\right]+\frac{\omega_{s}}{\omega_{i}} r_{i} I_{m 1} \cos \varphi_{1} \sin \left(\omega_{i} t\right)+\frac{V_{s}}{\omega_{i} L_{i}} \sin \left(\omega_{i} t\right)+i_{f}(0), \\
\frac{V_{s}\left(t-T_{s}\right)}{L_{i}}+i_{f}(0), & 0 \leq t<\frac{T_{s}}{2} \\
& \frac{T_{s}}{2} \leq t<T_{s} .
\end{array}\right.
$$


From Eq. 1, Eq. 2, Eq. 4 and Eq. 9, the current through $L_{i}$ at the instant $t=0$ is

$$
i_{f}(0)=\frac{V_{s}\left(\cos \frac{\pi \omega_{i}}{\omega_{s}}-1\right)}{Z_{i} \sin \frac{\pi \omega_{i}}{\omega_{s}}}+\frac{P_{o}}{V_{o}}-\left(\frac{\omega_{s}^{2}}{\omega_{i}^{2}} r_{i}-1\right) I_{m 1} \sin \varphi_{1}+\frac{\omega_{s} r_{i} I_{m 1} \cos \varphi_{1}\left(1+\cos \frac{\pi \omega_{i}}{\omega_{s}}\right)}{\omega_{i} \sin \frac{\pi \omega_{i}}{\omega_{s}}}
$$

After a short transient process, the inverter will be in a periodical steady state. Therefore, the current through $L_{i}$ is periodical. That is to say,

$$
i_{f}\left(T_{s}\right)=i_{f}(0)
$$

After Eq. 13 is substituted into Eq. 10, an expression is obtained as follows:

$$
\int_{0}^{T_{s}}\left[V_{s}-v_{r}(t)\right] d t=0
$$

Then, Eq. 9 is substituted into Eq. 14, the following result can be obtained and that is

$$
L_{i}=\frac{-V_{s}}{2 r_{i} I_{m 1} \sin \varphi_{1}}\left[\frac{\pi}{\omega_{s}}+\frac{\sin \frac{\pi \omega_{i}}{\omega_{s}}}{\omega_{i}}+\frac{\left(1-\cos \frac{\pi \omega_{i}}{\omega_{s}}\right)^{2}}{\omega_{i} \sin \frac{\pi \omega_{i}}{\omega_{s}}}\right]
$$

In addition, for the inverter, input energy is equal to output energy all the time in a stable period, that is

$$
P_{o}=\frac{1}{T_{s}} \int_{0}^{T_{S}} V_{s} i_{f}(t) d t
$$

Then, Eq. 11 and Eq. 12 are substituted into Eq. 16, a result can be obtained as follows:

$$
L_{i}=\frac{V_{s}\left(A_{1}+A_{2}\right)}{\frac{2 \pi}{\omega_{s}} P_{o}\left(\frac{1}{V_{s}}-\frac{1}{V_{o}}\right)+I_{m 1}\left(B_{1}-B_{2}\right)}
$$

where

$$
\begin{aligned}
& A_{1}=\frac{\cos \frac{\pi \omega_{i}}{\omega_{s}}-1}{\omega_{i}}\left(\frac{\sin \frac{\pi \omega_{i}}{\omega_{s}}}{\omega_{i}}+\frac{\pi}{\omega_{s} \sin \frac{\pi \omega_{i}}{\omega_{s}}}\right), \quad A_{2}=\frac{1-\cos \frac{\pi \omega_{i}}{\omega_{s}}}{\omega_{i}^{2}}-\frac{\pi^{2}}{2 \omega_{s}^{2}}, \\
& B_{1}=\frac{\omega_{s} r_{i} \cos \varphi_{1}\left(\cos \frac{\pi \omega_{i}}{\omega_{s}}-1\right)}{\omega_{i}^{2}}+\frac{r_{i}}{\omega_{s}}\left(2 \cos \varphi_{1}-\pi \sin \varphi_{1}\right), \\
& \text { and } B_{2}=\frac{2 \pi \sin \varphi_{1}}{\omega_{s}}\left(1-\left(\frac{\omega_{s}}{\omega_{i}}\right)^{2} r_{i}\right)+\frac{\omega_{s} r_{i} \cos \varphi_{1}\left(\cos \frac{\pi \omega_{i}}{\omega_{s}}+1\right)}{\omega_{i} \sin \frac{\pi \omega_{i}}{\omega_{s}}}\left(\frac{\sin \frac{\pi \omega_{i}}{\omega_{s}}}{\omega_{i}}+\frac{\pi}{\omega_{s}}\right) .
\end{aligned}
$$

From Eq. 15 and Eq. 17, the values of the inductor $L_{i}$ and $I_{m 1}$ can be solved. Furthermore, the capacitance $C_{i}$ can be determined from

$$
C_{i}=\frac{1}{\omega_{i}^{2} L_{i}}
$$




\section{Design Method of Resonant Rectifier}

For the equivalent rectifier shown in Fig. 2(b), the diode $D_{1}$ and $C_{r}$ are connected in parallel. There is an advantage for that. It can compensate for the adverse influence caused by the junction capacitance of the rectifier diode $D_{1}$. Actually, the designed capacitance consists of the junction capacitance of $D_{1}$ and the capacitance of $C_{r}$. Since the output DC source (the capacitor $C_{o}$ ) acts as an AC short, the rectifier circuit can be effectively transformed into the circuit shown in Fig. 3. In accordance with the second consumption in section II, as the input voltage, $v_{i}$ is defined as

$$
v_{i}(t)=V_{m 1} \sin \left(\omega_{s} t+\theta_{1}\right)+V_{s}
$$

where $V_{s}$ is the input voltage of the converter, $\omega_{s}$ is the operating frequency which is equal to the switching frequency of the converter, $V_{m 1}$ and $\theta_{1}$ represent the fundamental amplitude and phase of the voltage across $C_{i}$, respectively.

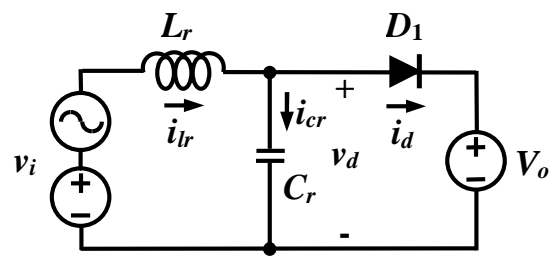

Fig. 3 Transformed circuit of the rectifier.

The operation period of the rectifier is made up of two states, off state and on state. However, it is unknown when the rectifying diode is turned off and on. Therefore, it is required to determine the instants $t_{\text {off }}$ and $t_{\text {on }}$ when the diode $D_{1}$ begins to be turned off and on.

While the rectifying diode $D_{1}$ is off, that is the time period $t_{\text {off }} \leq t<T_{s}+t_{\mathrm{on}}$, being similar to the analysis for the inverter in section III, there are a circuit constraint and two component constraints which can be taken advantage of, they are

$$
\begin{aligned}
& i_{l r}=i_{d}+i_{c r} \\
& v_{i}(t)-v_{d}(t)=L_{r} \frac{d i_{l r}(t)}{d t} \\
& i_{c r}(t)=C_{r} \frac{d v_{d}(t)}{d t}
\end{aligned}
$$

From Eq. 20 - Eq. 22, a second-order differential equation describing the voltage $v_{d}(t)$ can be represented with the following form:

$$
L_{r} C_{r} \frac{d^{2} v_{d}(t)}{d t^{2}}+v_{d}(t)=V_{s}+V_{m 1} \sin \left(\omega_{s} t+\theta_{1}\right)
$$

For this equation, its solution can be represented as

$$
v_{r}(t)=B_{1} \sin \left(\omega_{r} t\right)+B_{2} \cos \left(\omega_{r} t\right)+V_{s}+\frac{V_{m 1}}{1-\omega_{s}^{2} L_{r} C_{r}} \sin \left(\omega_{s} t+\theta_{1}\right)
$$

where

$$
\omega_{r}=\frac{1}{\sqrt{L_{r} C_{r}}},
$$

$B_{1}$ and $B_{2}$ are unknown and constant. Therefore, two initial conditions are required to determine these constants. At the instant $t_{\text {off }}$, for the rectifier, two initial conditions can be taken advantage of, the 
voltage across the capacitor $C_{r}, v_{d}\left(t_{\text {off }}\right)$ and the current through the capacitor $C_{r}, i_{l f}\left(t_{\text {off }}\right)$ are known and their values are zero. So, $B_{1}$ and $B_{2}$ can be solved. While the diode $D_{1}$ is on, the voltage across the capacitor $C_{i}$ is always equal to $V_{o}$. In the end, the voltage $v_{d}(t)$ can be represented as

$$
v_{d}(t)=\left\{\begin{array}{lr}
\left(r_{r} V_{m 1} \sin \left(\omega_{s} t_{\mathrm{off}}+\theta_{1}\right)+V_{o}-V_{s}\right) \cos \left(\omega_{r}\left(t-t_{\mathrm{off}}\right)\right)-r_{r} V_{m 1} \sin \left(\omega_{s} t+\theta_{1}\right)+V_{s} \\
+\frac{\omega_{s}}{\omega_{r}} r_{r} V_{m 1} \cos \left(\omega_{s} t_{\mathrm{off}}+\theta_{1}\right) \sin \left(\omega_{r}\left(t-t_{\mathrm{off}}\right)\right) & t_{\mathrm{off}} \leq t<T_{s}+t_{\mathrm{on}} \\
V_{o}, & t_{\mathrm{on}} \leq t<t_{\mathrm{off}} .
\end{array}\right.
$$

where

$$
r_{r}=\frac{1}{\left(\omega_{s} / \omega_{r}\right)^{2}-1} .
$$

In this equation, it is required to determine and solve four unknown variables, and they are $t_{\mathrm{off}}, t_{\mathrm{on}}, L_{r}$ and $C_{r}$ respectively. Four conditions or constraints are required to solve these unknown parameters.

When the diode $D_{1}$ is turned on, the voltage $v_{d}(t)$ is held at $V_{o}$, that is

$$
v_{d}\left(T_{s}+t_{\mathrm{on}}\right)=V_{o}
$$

Eq. 26 is substituted into Eq. 25, as a result,

$$
\begin{aligned}
& r_{r} V_{m 1} \sin \left(\omega_{s} t_{\text {off }}+\theta_{1}\right) \cos \left(\omega_{r}\left(\frac{2 \pi}{\omega_{s}}+t_{\text {on }}-t_{\text {off }}\right)\right)+\left(V_{o}-V_{s}\right)\left[\cos \left(\omega_{r}\left(\frac{2 \pi}{\omega_{s}}+t_{\text {on }}-t_{\text {off }}\right)\right)-1\right] \\
& +\frac{r_{r} \omega_{s}}{\omega_{r}} V_{m 1} \cos \left(\omega_{s} t_{\text {off }}+\theta_{1}\right) \sin \left(\omega_{r}\left(\frac{2 \pi}{\omega_{s}}+t_{\text {on }}-t_{\text {off }}\right)\right)-r_{r} V_{m 1} \sin \left(\omega_{s} t_{\text {on }}+\theta_{1}\right)=0
\end{aligned}
$$

The current through $L_{r}$ is periodical. Therefore,

$$
i_{l r}\left(T_{s}+t_{\text {on }}\right)=i_{l r}\left(t_{\text {on }}\right)
$$

From Eq. 21, during the time period $t_{\mathrm{on}} \leq t<t_{\mathrm{off}}$, the current through $L_{r}$ can be represented as

$$
i_{l r}(t)=\int_{t_{\mathrm{on}}}^{t}\left[v_{i}(t)-v_{d}(t)\right] d t+i_{l r}\left(t_{\mathrm{on}}\right)
$$

During the time period $t_{\mathrm{off}} \leq t<T_{s}+t_{\mathrm{on}}$, the current through $L_{r}$ is

$$
i_{l r}(t)=\int_{t_{\mathrm{off}}}^{t}\left[v_{i}(t)-v_{d}(t)\right] d t+i_{l r}\left(t_{\mathrm{off}}\right)
$$

Substituting Eq. 28 into Eq. 29 results in

$$
\int_{t_{\mathrm{on}}}^{T_{S}+t_{\mathrm{on}}}\left[v_{i}(t)-v_{d}(t)\right] d t=0
$$

Then, Eq. 25 is substituted into Eq. 31, the following result can be obtained and that is

$$
\begin{aligned}
& \frac{r_{r}}{\omega_{r}} V_{m 1} \sin \left(\omega_{s} t_{\text {off }}+\theta_{1}\right) \sin \left(\omega_{r}\left(\frac{2 \pi}{\omega_{s}}+t_{\text {on }}-t_{\text {off }}\right)\right)+\frac{\left(V_{o}-V_{s}\right)}{\omega_{r}} \sin \left(\omega_{r}\left(\frac{2 \pi}{\omega_{s}}+t_{\text {on }}-t_{\text {off }}\right)\right) \\
& +\left(V_{o}-V_{s}\right)\left(t_{\text {on }}-t_{\text {off }}\right)+\frac{r_{r} \omega_{s}}{\omega_{r}^{2}} V_{m 1} \cos \left(\omega_{s} t_{\text {off }}+\theta_{1}\right)\left[1-\cos \left(\omega_{r}\left(\frac{2 \pi}{\omega_{s}}+t_{\text {on }}-t_{\text {off }}\right)\right)\right]
\end{aligned}
$$




$$
+\frac{r_{r} V_{m 1}}{\omega_{s}}\left[\cos \left(\omega_{s} t_{\text {on }}+\theta_{1}\right)-\cos \left(\omega_{s} t_{\text {off }}+\theta_{1}\right)\right]=0
$$

The values of the instants $t_{\mathrm{on}}$ and $t_{\mathrm{off}}$ can be solved from Eq. 27 and Eq. 32 .

While the diode is $D_{1}$ on, from Eq. 29, the current through $L_{r}$ is solved as follows:

$$
i_{l r}(t)=\frac{1}{L_{r}}\left\{\frac{V_{m 1}}{\omega_{s}}\left[\cos \left(\omega_{s} t_{\text {off }}+\theta_{1}\right)-\cos \left(\omega_{s} t+\theta_{1}\right)\right]+\left(V_{s}-V_{o}\right)\left(t-t_{\text {off }}\right)\right\}
$$

Additionally, the rectifier delivers and outputs its energy only from the instant $t_{\text {on }}$ to the instant $t_{\text {off. }}$. Hence,

$$
P_{o}=\frac{1}{T_{S}} \int_{t_{\mathrm{on}}}^{t_{\mathrm{off}}} V_{o} i_{l r}(t) d t
$$

Then, Eq. 29 is substituted into this equation, the value of the inductor $L_{i}$ is obtained from

$$
\begin{aligned}
L_{r}= & \frac{V_{o}}{2 \pi P_{o}}\left\{V_{\mathrm{AC}}\left[\cos \left(\omega_{s} t_{\mathrm{off}}+\theta_{1}\right)\left(t_{\mathrm{off}}-t_{\mathrm{on}}\right)\right]+\frac{\sin \left(\omega_{s} t_{\mathrm{on}}+\theta_{1}\right)-\sin \left(\omega_{s} t_{\mathrm{off}}+\theta_{1}\right)}{\omega_{s}}\right\} \\
& +\frac{1}{2} \omega_{s}\left(V_{o}-V_{S}\right)\left(t_{\mathrm{off}}-t_{\mathrm{on}}\right)^{2}
\end{aligned}
$$

Furthermore, the capacitance $C_{r}$ can be determined from

$$
C_{r}=\frac{1}{\omega_{r}^{2} L_{r}}
$$

\section{Simulation Results}

The proposed design method in this paper is validated with a numerical design example. The design example is simulated in MATLAB and SABER respectively. The results of two simulations are quite the same. The output power of the converter is $8.0 \mathrm{~W}$, the input voltage and the output voltage are $12 \mathrm{~V}$ and $24 \mathrm{~V}$ respectively. The switching frequency is set to $50 \mathrm{MHz}$. For the inverter, the resonant frequency of the $L C$ tank is $45 \mathrm{MHz}$. While for the rectifier, the resonant frequency of the $L C$ tank is 51.03 MHz. In addition, the outphasing angle $\varphi_{1}$ is set to -1.1 radians. In accordance with these design conditions, the proposed design method and design formulae, the components $L_{i}, C_{i}, L_{r}$ and $C_{r}$ in Fig. 1 are designed and their design values are listed in Table 1.

Table 1 List of Components

\begin{tabular}{|c|c|}
\hline Component & Design Value \\
\hline$L_{i}$ & $81.45 \mathrm{nH}$ \\
\hline$C_{i}$ & $153.57 \mathrm{pF}$ \\
\hline$L_{r}$ & $111.67 \mathrm{nH}$ \\
\hline$C_{r}$ & $87.11 \mathrm{pF}$ \\
\hline
\end{tabular}

Fig. 4 shows the voltage across $C_{i}$, the voltage across $C_{r}$, the current through $L_{i}$ and the current through $L_{r}$. From Fig. 4(a), at the instants $t=(k+1 / 2) T_{s}, k=0,1,2$, , the voltage across $C_{i} v_{r}(t)$ is closely equal to zero, that is to say, ZVS occurs. That demonstrates the switch of the inverter achieves soft switching, when it is turned on and off. Besides, from Fig. 4(b), the current through $L_{r} i_{l r}(t)$ is nearly sinusoidal and there is a DC offset. The result shows the definition for $i_{r}(t)$ in Eq. 1 is reasonable 
and the simplification analysis for the converter is suitable and acceptable. From Fig. 4(a), at the instants $t=(k+1 / 2) T_{s}, k=0,1,2$, ; the voltage across $C_{i} v_{r}(t)$ is closely equal to zero, that is to say, ZVS occurs. That demonstrates the switch of the inverter achieves soft switching, when it is turned on and off. Besides, from Fig. 4(b), the current through $L_{r} i_{l r}(t)$ is nearly sinusoidal and there is a DC offset. The result shows the definition for $i_{r}(t)$ in Eq. 1 is reasonable and the simplification analysis for the converter is suitable and acceptable.

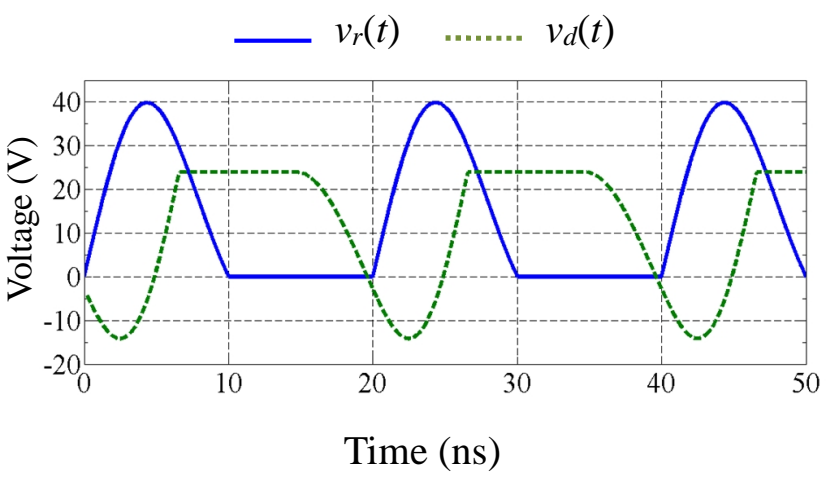

(a) Voltage across $C_{i}$ and $C_{r}, v_{r}(t)$ and $v_{d}(t)$.

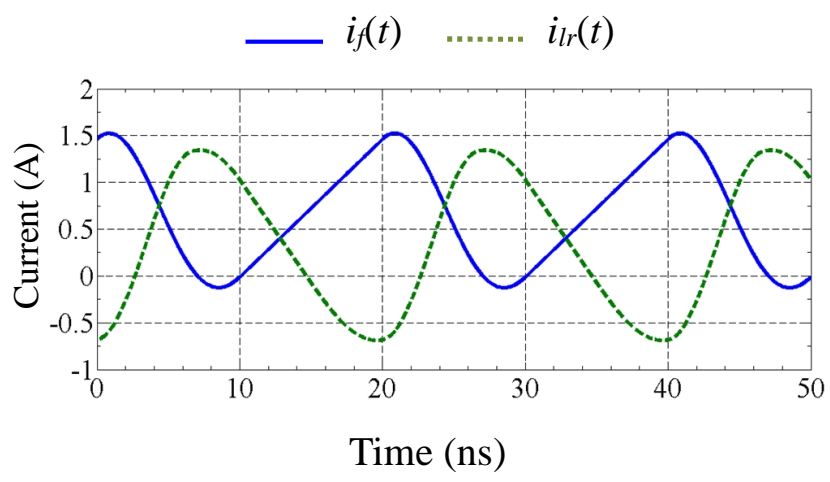

(b) Current through $L_{i}$ and $L_{r}, i_{f}(t)$ and $i_{l r}(t)$.

Fig. 4 Voltage across $C_{i}$ and $C_{r}$, current through $L_{i}$ and $L_{r}$.

\section{Conclusion}

This paper represents an approach suitable for the design of a ZVS DC/DC converter switched at very high frequencies in detail. As the converter employs a resonant rectifier, it can be divided into two independent parts, which can be designed respectively. This method is applied in a numerical design example. The designed converter is operated at $50 \mathrm{MHz}$. To verify the converter and the design method, the design example is simulated in MATLAB and SABER respectively. The simulation waveforms closely accord with the results derived from the analysis. in addition, the simulation results demonstrate that the converter implements ZVS very well and the simplification analysis for the converter is suitable and feasible.

\section{References}

[1] J. M. Burkhart, R. Korsunsky, and D. J. Perreault: Design methodology for a very high frequency resonant boost converter, in Proc. Int. Power Electron. Conf., Jun. 2010, p. 1902-1909.

[2] M. K. Kazimierczuk and D. Czarkowski: Resonant Power Converters, 2nd ed. (John Wiley \& Sons, New York 2011)

[3] M. M. Jovanovic: Merits and limitations of resonant and soft-switched converters, in Proc. Telecommun. Energy Conf., 1992, p. 51-58.

[4] M. K. Kazimierczuk: Analysis of class E zero-voltage-switching rectifier, IEEE Trans. Circuit Syst., vol. 37, no. 6, p. 747-755, Jun. 1990.

[5] A. Ivascu, M. K. Kazimierczuk, and S. Birca-Galateanu: "Class E resonant low $d v / d t$ rectifier," IEEE Trans. Circuits Syst. I, vol. 39, no. 8, p. 604-613, Aug. 1992. 
[6] W. A. Nitz, W. C. Bowman, F. T. Dickens, F. M. Magalhaes, W.Strauss, W. B. Suiter, and N. G. Ziesse: A new family of resonant rectifier circuits for high frequency DC-DC converter applications, in Proc. Appl. Power Electron. Conf. Expo., 1988, p. 12-22.

[7] M. K. Kazimierczuk: Class E low $d v_{D} / d t$ rectifier, IEE Proc. B Electric Power Appl., vol. 136, no. 6, p. 257-262, Nov. 1989.

[8] M. K. Kazimierczuk: Analysis of class E zero-voltage-switching rectifier, IEEE Trans. Circuit Syst., vol. 37, no. 6, p. 747-755, Jun. 1990.

[9] R. C. Pilawa-Podgurski, A. D. Sagneri, J. M. Rivas, D. I. Andersion, and D. J. Perreault: Very high frequency resonant boost converters, IEEE Trans. Power Electron., vol. 24, no. 6, p. 1654-1665, Jun. 2009.

[10]S. Birca-Galateanu and J.-L. Cocquerelle: Class E half-wave low $d v / d t$ rectifier operating in a range of frequencies around resonance, IEEE Trans. Circuits Syst.-I: Fundam. Theory Appl., vol. 42, no. 2, p. 83-94, Feb. 1995.

[11]J. Rivas, D. Jackson, O. Leitermann, A. Sagneri, Y. Han, and D. Perreault: Design considerations for very high frequency DC-DC converters, in Proc. 37th IEEE Power Electron. Spec. Conf., Jun. 18-22, 2006, p. 1-11.

[12]W. A. Tabisz and F. C. Lee: DC analysis and design of zero-voltage switched multi-resonant converters, in Proc. Power Electron. Spec. Conf., Jan. 1989, vol. 1, p. 243-251.

[13]J. A. Santiago-Gonzalez, K. M. Elbaggari, K. K. Afridi, and D. J. Perreault: Design of class E resonant rectifiers and diode evaluation for VHF power conversion, IEEE Trans. Power Electron., vol. 30, no. 9, p. 4960-4972, Sept. 2015. 